\title{
Improving Uptime of Femtocell Deployments in Rural and Remote Regions using Power Spreading
}

\author{
Amit Kumar Vishwakarma \\ NRI Institute of Information Science \& Technology \\ Department of Electronics and Communication \\ Bhopal, Madhya Pradesh, India
}

\author{
Akhilesh Jain \\ NRI Institute of Information Science \& Technology \\ Department of Electronics and Communication \\ Bhopal, Madhya Pradesh, India
}

\begin{abstract}
Small cells such as Femtocell have proved to be a cost effective solution to improve cellular connectivity in enterprise and residential deployments. Even though femtocells are inherently energy efficient, their operation in grid energy deprived rural and remote regions will require significant improvement in energy efficiency. Rural and remote are characterized by low mobile user density/data demands and hence more attention should be given on improving the energy efficiency and network uptime rather than on maximizing the system throughput. In this paper, we consider a rural femtocell network powered by solar cells and battery subsystem. The energy consumption of a femtocell is dependent upon the number of mobile users associated with it. Keeping this in mind, we develop an efficient spectrum and power allocation scheme called power spreading which when used with Max Reference Signal Received Power (RSRP) based cell selection scheme results in a significant improvement in network uptime. Additionally, this scheme has shown to improve the energy efficiency of the network without compromising the mobile users' bitrates. The obtained results are verified using extensive simulations.
\end{abstract}

\section{Keywords}

Femtocell, Cell Selection Scheme, Reference Signal Received Power, Power Spreading, Network Uptime.

\section{INTRODUCTION}

Future wireless networks require ubiquitous connectivity with high data rates to improve the quality of service (QoS) of mobile users [1]. To handle exponential increasing demands for high bitrate and ubiquitous connectivity, mobile operators are looking for dense deployment of miniature cellular base stations inside users' homes/offices. Deployments of such low-cost, low-powered cellular base stations at close proximity to mobile users not only improve network throughput, coverage, and reliability but also enhance the life of battery-operated mobile devices. These small base stations are more commonly known as Femtocell or Femto Access Points (FAPs) or simply Femtocells [2].

Dense deployment of FAPs allows efficient reuse of available wireless spectrum. This leads to improvement in the system capacity manifold without increasing additional deployment and maintenance costs to the cellular operators. Additionally, it also helps in solving indoor coverage problem as indoor users are severely affected by wall penetration losses. The unique benefit of femtocell technology is that users require no new equipment. Any existing mobile user can connect to and get serviced by FAPs. Additionally, the deployment and maintenance costs of the femtocell are very low which make it feasible for both home users and cellular operators. Indeed, a well-designed femtocell/macrocell-integrated network can divert huge amounts of traffic from congested and expensive macro-cellular networks to femto-cellular networks. From the wireless operator point of view, the ability to offload a large amount of traffic from macro-cellular networks to femtocells is the most important advantage of the femtocell/macrocellintegrated network architecture [3]. This will not only reduce the investment capital, maintenance expenses, and operational costs but will also improve the reliability and energy efficiency of the cellular networks [4].

Use of low-cost low-power FAPs for providing cellular coverage in rural and remote regions has grasped the attention of industry and academia [5]. However, $24 \times 7$ availability of grid power for femtocell operation in rural and remote regions poses a serious challenge. An alternative approach is the use of renewable energy resources such as solar and wind energy. In rural and remote areas, the count of User Equipments (UEs) and their data rate demands are low. Consequently, most FAPs remain idle but still consume some finite energy for their operation. Hence, to serve more UEs and for longer durations, it is of utmost importance to improve FAPs' uptime by reducing their energy consumption. The motivation behind using FAPs to improve network performance is their ability to efficiently reuse the available wireless spectrum [2]. The inherent low transmission power capability of FAPs keeps cochannel interference to minimum. The traditional spectrum and power allocation technique divides the available bandwidth into a number of logical subchannels and assigns equal transmit power to them. These subchannels are then assigned to UEs to satisfy their data demands. This approach is rather inefficient as it may results in under-utilization of subchannels at FAPs, specially when UE count and/or data demands are low. Additionally, the chances of experiencing co-channel interference are high when femtocells have overlapping coverage.

In this paper, we aim at improving the performance of femtocell network in rural and remote regions by improving the underlying cell selection scheme used for assigning the UEs to FAPs. We propose a power spreading based spectrum and power allocation scheme which is used in combination with Max RSRP cell selection scheme to improve uptime of femtocell network [6]. We suggest that instead of assigning only required number of subchannels, all available subchannels should be assigned to UEs with each FAP. Additionally, the transmit power of each subchannel should be reduced to the minimum possible value. This in turn improves the spectrum utilization, and lowers the femtocell energy consumptions and co-channel interference. Our proposed technique is shown to improve the energy efficiency of the network too. Such a solution will help in accelerating the deployment and maintenance of femtocells powered using solar cells and battery subsystem in cellular coverage deprived regions. 


\section{CELL SELECTION SCHEME}

Cell Selection scheme defines the criterion on which an incoming UE choose a base station for communication. These criteria may include reference signal strength, reference signal quality, SINR, or even expected bitrate. The most widely used cell selection scheme in the literature is Max RSRP scheme. This scheme assigns the incoming UE to a base station which provides them with the highest signal strength [7]. Hence, UE $u$ get assigned to femtocell base station $f$ if,

$$
\text { CellID }_{u}=\arg _{f} \operatorname{Max}\left(\operatorname{RSRP}_{f}\right)
$$

This cell selection scheme usually ends up assigning UEs to their nearest femtocells. However, this scheme has a drawback that it does not consider the system load and energy consumption in the cell selection criteria. Hence, even though Max RSRP provides UEs the best signal strength, it may end up lowering the energy efficiency.

An enhancement to Max RSRP cell selection scheme is the use of cell biasing. Cell biasing allows us to offload higher number of UEs to low power FAPs by adding a positive Range Expansion Bias (REB) to the RSRP from FAPs [8]. Note that, this REB does not change the transmit power of FAPs, but still offloads UEs to FAPs by changing the user association criteria. Cell biasing scheme is mainly used in heterogeneous cellular networks where deployments of base stations having different transmit power is done. Due to difference in transmit power, Max RSRP scheme ends up assigning more UEs to high power macrocells and hence keeping FAPs underutilized. However, if a network setup has base stations with similar transmit power (such as our rural femtocell deployment), use of same REB with Max RSRP for all base stations will not provide any benefits.

\section{SPECTRUM AND POWER ALLOCATION TECHNIQUE}

Once UEs are assigned to FAPs using any cell selection scheme, resources such as spectrum and transmit power need to be distributed among them. The traditional spectrum and power allocation technique distributes the maximum transmit power equally among all the available subchannels. Then, based on UEs' bitrate requests, required number of subchannels are assigned to them [6]. This scheme has various disadvantages. First, it uses the maximum possible transmit power in each subchannel which in turn gives rise to cochannel interference to neighboring femtocell UEs. Second, due to high transmit power, the energy consumption of femtocell also increases. This will consequently lower the energy efficiency and network uptime of FAP in grid energy deprived solar powered FAPs.

\section{SYSTEM MODEL}

Our system model consists a set of $F$ solar powered FAPs $(f=$ 1 ...F) deployed in a geographical region as shown in Figure 1. FAPs are assumed to operate in "Open Access" mode in order to serve any UEs in their vicinity. Max RSRP based cell selection scheme is used to assign UEs to FAPs. Each FAP is equipped with solar cells and necessary hardware such as charge controller and battery subsystem. Each FAP is always in ACTIVE state to serve its UEs unless its battery subsystem capacity falls below a threshold value, at which point FAP shifts to SLEEP mode. A total of $U$ UEs are distributed uniformly. The downlink bitrate requested by UE $u$ is represented by $\beta_{u}$. The bandwidth at each base station is divided into $N$ subchannels each $W$ hertz wide. The number of subchannels assigned to UE $u$ is represented by $N_{u}$.

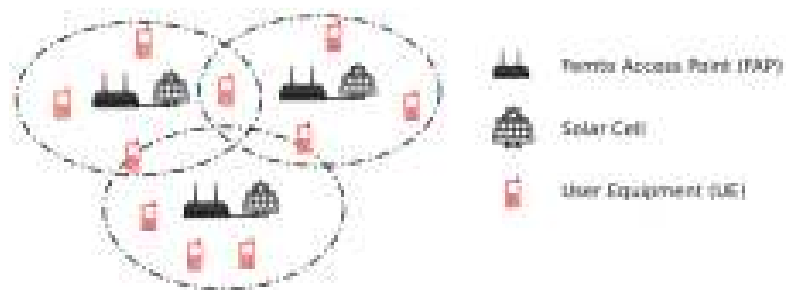

Fig 1: Solar Powered Femtocell Deployment

Let $T_{f, s}^{u}$ represent the instantaneous subchannel transmit power of FAP $f$ for UE $u$ over downlink subchannel $s$. Considering Full Frequency Reuse scheme (FFR) among FAPs, the instantaneous downlink SINR of UE $u$ is given by,

$$
\Gamma_{f, s}^{u}=\frac{T_{f, s}^{u} G_{f}^{u}}{\sum_{F} T_{l, s}^{u} H_{l, s}^{u}+W N_{0}}
$$

where $G_{f}^{u}\left(H_{f}^{u}\right)$ is the effective signal (interference) gain to UE u from FAP $f$. $N_{0}$ is the noise spectral density. Based on the SINR calculated by Equation 1, the average bitrate obtained by UE $u$ from FAP $f$ is calculated as [9],

$$
B R_{u}^{f}=N_{u} * W * \log _{2}\left(1+\Gamma_{f, s}^{u}\right) \quad \text { bits/sec }
$$

\subsection{Energy Generation, Energy Consumption, and Energy Efficiency}

Energy consumption of a FAP is taken to be load dependent with some fixed "Zero Load" consumption. The instantaneous energy consumption of FAP $f$ is calculated using the following equation $[10]$,

$$
E_{f}=E_{f}^{0}+\frac{\kappa_{f}}{\varsigma_{\mathrm{f}}}+\psi_{f}
$$

where $E_{f}^{0}$ is fixed "Zero Load" power. $\varsigma_{\mathrm{f}}$, and $\psi_{f}$ represent the power amplifier efficiency and signal processing overhead, respectively. Here $\kappa_{f}$ is total input power to transmitting antenna obtained by summing up transmit power of all the downlink subchannels in use $\left(\kappa_{f}=\sum_{s} T_{f}\right)$.

Consider FAP $f$ in the $i^{\text {th }}$ time slot. The state of its battery is indicated by variable ${ }^{B}{ }_{f, i}$, which represents the amount of energy stored in it at the $i^{t h}$ time slot. The charging profile of battery is given by the variable $C_{f, i}$ which determines the amount of solar energy FAP $f$ 's battery will receive. Hence, the battery state at $(i+1)^{\text {th }}$ time slot can be calculated as,

$B_{f,(i+1)}=B_{f, i}+C_{f, i}-E_{f}$

To compare energy efficiency performance of different cell selection schemes, we take Energy Consumption Rating (ECR) as performance metric [11]. ECR is the ratio of total 
energy consumed to total system capacity. ECR can be calculated as,

$$
\text { ECR(watts / Mbps) }=\frac{\text { Energy Consumptio } n}{\text { System Capacity }}
$$

Hence, lower the ECR, more energy efficient the system will be.

\section{PROBLEM FORMULATION AND SOLUTION DESCRIPTION}

\subsection{Optimization Problem}

The objective of our work is to improve the uptime of FAPs deployed in a geographical region by improving the spectrum and power allocation for Max RSRP cell selection scheme. For this, we first assign UEs to FAPs and then focus on increasing the energy stored in the batteries by reducing the overall FAPs' energy consumption. Thus, we formulate our optimization problem as follows,

$\max \sum_{f} \sum_{i} B_{f, i}$

such that $B_{f, i} \geq 0, \forall f, i$ and $B R_{u}^{f} \geq \beta_{u} \forall u$

\subsection{Power Spreading Spectrum and Power Allocation Technique}

The traditional spectrum and power allocation technique equally distributes the maximum transmit power among all subchannels. Then, based on SINR, UEs are assigned the required number of subchannels to satisfy their bitrate demands. For example, UE 1 and UE 2 in Figure 2(a) are allocated one and two subchannels (SCh), respectively, to satisfy their bitrate demands. However, subchannel 4 and 5 are free with zero transmit power allocation. Such uneven power allocation over subchannels results in increased frequency selective interference for neighboring femtocell users. Our spreading based spectrum and power allocation techniques assigns all available subchannels to UEs with lowest possible subchannel transmit power [12]. For example, in Figure 2(b), all 5 subchannels are assigned to UE 1 and UE 2 with equal power to satisfy their bitrate demands. Note that, the transmit power over each subchannels is quite lower compared to the traditional approach.

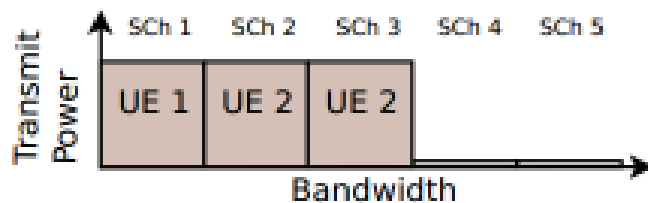

(a) Spectrum and power allocation : without spreading

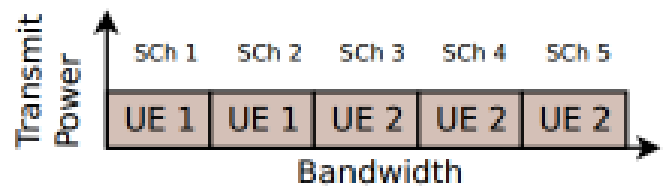

(b) Spectrum and power allocation : with spreading

Fig 2: Power Spreading

When an UE shifts from OFF state to ON state, it requests for data from its associated FAP. Since, there are no subchannels available, we try to free up some subchannels from existing UEs. For this, we use power spreading which iteratively increases the subchannel transmit power by a factor (say $\delta$ ) until enough number of subchannels are available for incoming UE. Similarly, when an UE shifts from ON state to OFF state, the subchannel transmit power is iteratively decreased to the minimum possible value whilst satisfying bitrate requests of remaining UEs.

\section{SIMULATION RESULTS}

Our simulation setup consists of 10 FAPs and 80 UEs deployed in the simulation region as shown in Figure 3. Discrete event simulations are done where UEs are assumed to follow an ON-OFF pattern with durations of ON and OFF period follow an exponential distribution with mean 5 and 120 minutes, respectively. Whenever, an UE is in ON state, it requests for data from its associated FAP. Bitrate requests of UEs vary uniformly between $256 \mathrm{Kbps}$ and $2 \mathrm{Mbps}$. The rest of the simulation parameters are summarized in Table. 1.

Table 1. Simulation Parameters

\begin{tabular}{|c|c|}
\hline Parameter & Value \\
\hline Bandwidth & $3 \mathrm{MHz}$ \\
\hline Number of Subchannels & 16 \\
\hline FAP Transmit Power & $23 \mathrm{dBm}$ \\
\hline UE Density & $100 \mathrm{UEs} / \mathrm{sq} \cdot \mathrm{km}$ \\
\hline Path Loss Coefficient & 3.5 \\
\hline Gaussian Noise Figure & $174 \mathrm{dBm} / \mathrm{Hz}$ \\
\hline Idle Mode FAP Energy Consumption & $5 \mathrm{Watts}$ \\
\hline
\end{tabular}

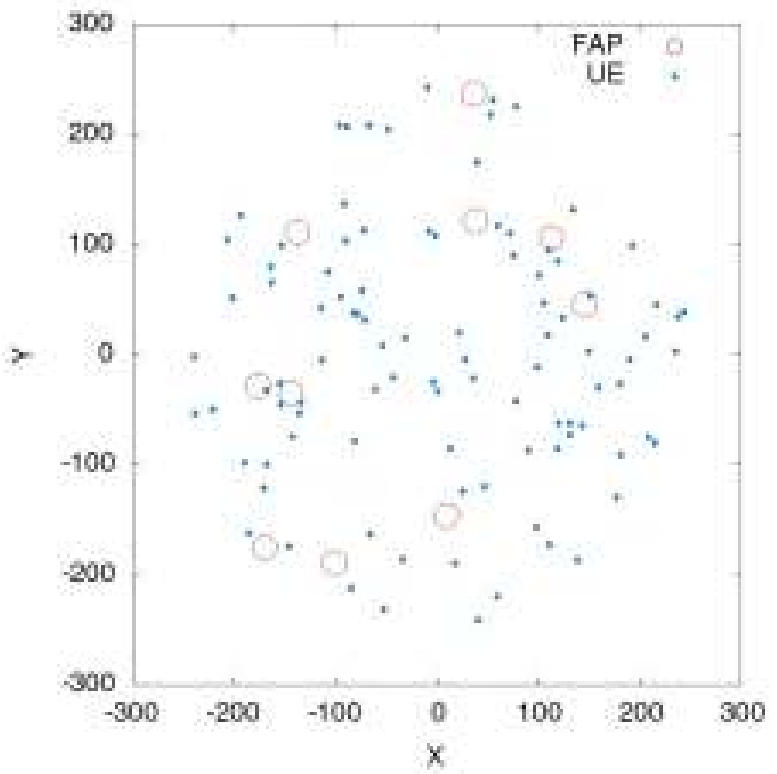

Fig 3: Simulation Scenario

\subsection{Performance Metrics}

System Throughput: System throughput is defined as the sum of downlink bitrate of all the UEs in the system.

Energy Consumption: Energy consumption is the sum of energy consumed by all the FAPs in the system

Uptime: Uptime is the fraction of total simulation for which an FAP remains in ACTIVE mode.

In order to analyze the performance of our proposed power spreading technique for Max RSRP cell selection scheme, we compare it with the traditional spectrum and power allocation 
technique where maximum transmit power is assigned to each FAP subchannel. Figure 4 represents the Cumulative Distribution Function (CDF) SINR of UEs associated with the FAPs for both traditional and power spreading based cell selection scheme. As can be seen that our proposed spreading technique when used with Max RSRP scheme result in a slight improvement in UEs SINR. This improvement is the result of using lower subchannel transmits power in FAPs to serve UEs, which in turn reduces the co-channel interference and the SINR of nearby UEs.

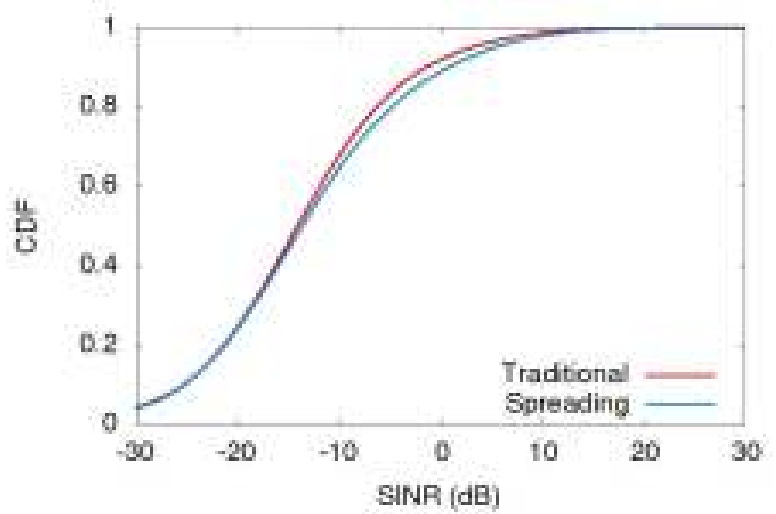

Fig 4: CDF of UEs SINR

Figure 5 represent the CDF bitrate of all the UEs in the system for both traditional and our proposed spreading technique. The CDF of both the techniques closely overlaps with each other. This is due to the fact that both of these techniques assign only the required number of resources (transmit power/subchannels) to satisfy the bitrate request of UEs.

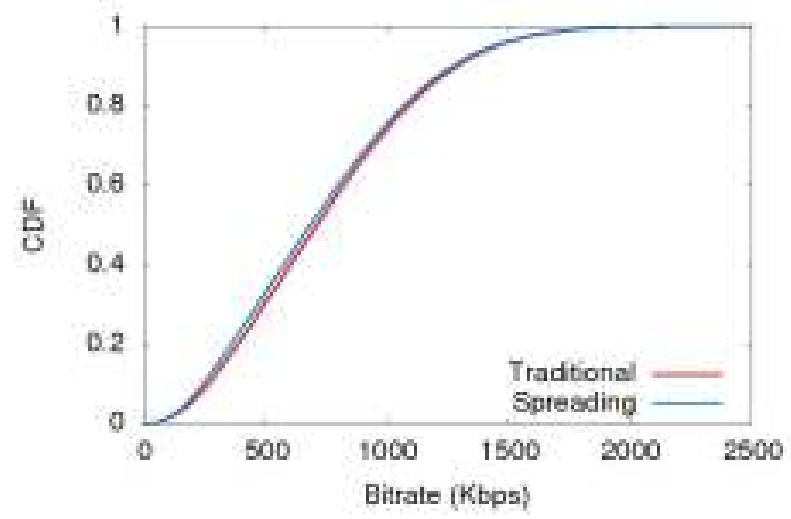

Fig 5: CDF of UEs Bitrate

The primary benefit of our proposed technique is on improving the uptime of solar powered FAPs as shown in Figure 6. Since our proposed technique uses the lowest transmit power, it also has the lowest energy consumption. Due to this, FAPs in the network remain in ACTIVE for longer duration of time and serve more number of UEs. This also results in a higher system throughout as shown in Table 2. Additionally, when this increase in system throughput is complemented by slightly lower energy consumption, leads to significant improvement in ECR (Table 2).

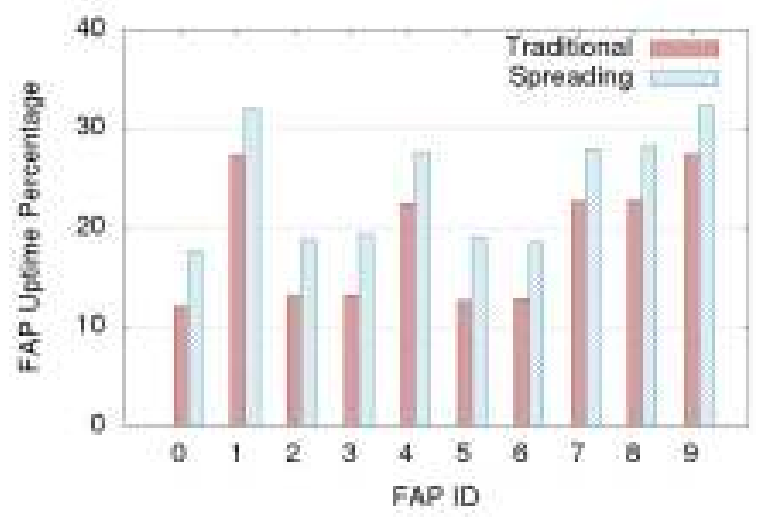

Fig 6: Uptime Percentage

Table 2. Throughput, Energy Consumption, and ECR

\begin{tabular}{|c|c|c|c|}
\hline & $\begin{array}{c}\text { Throughput } \\
\text { (Mbps) }\end{array}$ & $\begin{array}{c}\text { Energy } \\
\text { Consumption } \\
\text { (Watts) }\end{array}$ & $\begin{array}{c}\text { ECR } \\
\text { (Watts/Mbps) }\end{array}$ \\
\hline $\begin{array}{c}\text { RSRP: No } \\
\text { Spreading }\end{array}$ & 41.017 & 91.405 & 2.228 \\
\hline $\begin{array}{c}\text { RSRP: With } \\
\text { Spreading }\end{array}$ & 48.213 & 91.150 & 1.890 \\
\hline
\end{tabular}

\section{CONCLUSION}

Use of renewable power sources such as solar cell can facilitate cost affective femtocell deployment for sparsely populated rural and remote regions. We have suggested an improvement in Max RARP cell selection scheme by using power spreading in spectrum and power allocation for femtocell networks. Our proposed scheme has proved to improve the uptime of femtocells without affecting downlink data rates of mobile users. Additionally, improvement in network energy efficiency is also observed by the use of power spreading in femtocell network.

Energy consumption in cellular network is continuously increasing and hence it is of at most interest to look into the energy efficient deployment and maintenance of cellular networks. Along with femtocells and mobile users, the energy consumption of other components of cellular networks such as backhaul links and network routers/gateways should also be considered in the energy efficiency optimization criteria. Future work in this direction can incorporate the backhaul links' capacity and energy consumption into the cell selection and power spreading criteria. This not only improve the overall energy efficiency of the network but also distribute the user load among femtocells based on the capacity of the backhaul links.

\section{REFERENCES}

[1] "Cisco Visual Networking Index: Global Mobile Data Traffic Forecast Update, 2015-2020," Cisco, White Paper, February 2016.

[2] J. Andrews, H. Claussen, M. Dohler, S. Rangan, and M. Reed, "Femtocells: Past, present, and future," IEEE Journal on Selected Areas in Communications, vol. 30, no. 3, pp. 497-508, April 2012.

[3] A. K. Vishwakarma, A. Jain, and S. Jain, "Present and future research directions for cell selection in femtocell networks," International Journal of Research in Technology, vol. 2, no. 2, pp. 5-10, April 2016.

[4] A. K. Sharma, P. N. Suman, and A. Jain, "Improving 
energy efficiency of femtocell network by joint estimation of downlink throughput and uplink power," in Proceedings of the International Conference on Advanced Computing and Communication Systems, Jan 2015, pp. 1-5.

[5] 'Deployment issues for rural and remote small cells', Release 5, Document 156.05.01, Feb 2015. [Online]. Available:

http://www.scf.io/en/use_case_documents/Rural.php

[6] R. Thakur, V. J. Kotagi and C. S. R. Murthy, "An energy efficient cell selection scheme for femtocell network with spreading," Proceedings of the IEEE 26th Annual International Symposium on Personal, Indoor, and Mobile Radio Communications (PIMRC), Hong Kong, 2015, pp. 1569-1573.

[7] R. Thakur, S. Mishra, and C. R. Murthy, "A loadconscious cell selection scheme for femto-assisted cellular networks," in Proceedings of the IEEE 24th International Symposium on Personal Indoor and Mobile Radio Communications (PIMRC), Sept 2013,pp. 23782382 .

[8] R. Thakur, R. Singh, C. S. R. Murthy, An energy efficient framework for user association and power allocation in HetNets with interference and rate-loss constraints, Computer Communications, Volume 94, 15 November 2016, Pages 57-71.

[9] E. C. Cherry, "A history of the theory of information," Proceedings of the IEE - Part III: Radio and communication engineering, vol. 98, no. 55, pp. 383393, September 1951.

[10] M. Deruyck, D. De Vulder, W. Joseph, and L. Martens, "Modelling the power consumption in femtocell networks," in Proceedings of the IEEE Wireless Communications and Networking Conference Workshops, April 2012, pp. 30-35.

[11] X. Wang, A. V. Vasilakos, M. Chen, Y. Liu, and T. T. Kwon, "A survey of green mobile networks: Opportunities and challenges," ACM Mobile Networks and Applications, vol. 17, no. 1, pp. 4-20, February 2012.

[12] R. Thakur, S. N. Swain, and C. S. R. Murthy, "An Energy Efficient Cell Selection Framework for Femtocell Networks with Limited Backhaul Link Capacity," IEEE Systems Journal, vol. PP, pp. 1-10, June 2017. 